Ann. Génét. Sél. anim., 1982, 14 (4), 417-434

\title{
Etude du profil génétique des ovins de l'Arc Alpin italien à l'aide de marqueurs à effets visibles
}

\author{
I. BONACINI *, J.-J. LAUVERGNE **, G. SUCCI *** et G. ROGNONI **** \\ * Associazione Italiana Allevatori, via G. Tomassetti 9, Rome (Italie) \\ ** I.N.R.A., Département de Génétique animale, C.N.R.Z., F 78350 Jouy-en-Josas \\ *** Facolta di Agraria, Istituto di Zootecnica Generale, Via Celoria 2, Milan (Italie) \\ **** Facolta di Veterinaria, Istituto di Zootecnica Generale, Via Celoria 10, Milan (Italie)
}

\section{Résumé}

L'Arc Alpin italien peut être divisé en 3 grandes régions naturelles : la plaine padane, les collines subalpines et la montagne proprement dite.

La plus grande partie de l'effectif ovin actuel (250 à 300000 têtes) est basée dans la zone collinaire et elle effectue des mouvements saisonniers entre la plaine padane (l'hiver) et la montagne (l'été).

Dans la montagne vivent des petites populations plus sédentaires dont l'effectif total se situe entre 100 et 150000 têtes.

Il n’y a plus d'ovins basés dans la plaine du Pô.

Au point de vue racial, on a distingué, d'après leurs effectifs et les mouvements de dispersion de leurs reproducteurs attestés depuis longtemps, quatre races influençantes, qui sont toutes collinaires : Delle Langhe, Biellese, Bergamasque et Lamon. Les autres races considérées (25 au total) étaient considérées comme influencées.

Un indice d'archaïsme, somme de 5 notes objectives d'archaïsme, a été établi. Les caractères considérés étaient le format, le profil fronto-nasal, la forme et la longueur des oreilles, la coloration des zones poilues et le cornage.

L'indice moyen d'archaïsme des races considérées comme influençantes était $i=1$. Celui des 25 races considérées comme influencées était $\bar{i}=4,32$.

Au cours de cette analyse, on s'est aperçu que le Delle Rocce (ou Tiroler Bergschaf) pouvait être considéré comme une race influençante.

L'indice d'archaïsme des races influencées croît à peu près linéairement lorsque l'on s`éloigne des races influençantes: en moyenne d'environ 1 point tous les $20 \mathrm{~km}$.

Ces races influençantes auraient été créées dès le $14^{\mathrm{e}}$ siècle pour produire de la laine requise par l'industrie lainière du Nord de l'Italie alors en plein essor, avec des critères externes (grand format, toison blanche, chanfrein busqué, longues oreilles pendantes) se démarquant des critères du mouton traditionnel anciennement établi. Leur succès incomplet s'explique par le maintien d'un élevage de subsistance dans les vallées alpines.

\section{Introduction}

L'organisation de la conservation des stocks génétiques animaux passe par la connaissance de leur distribution géographique. Celle-ci est soumise à un certain nombre de lois que l'on commence à entrevoir (LAUVERGNe, 1979 a, 1981, 1982). 

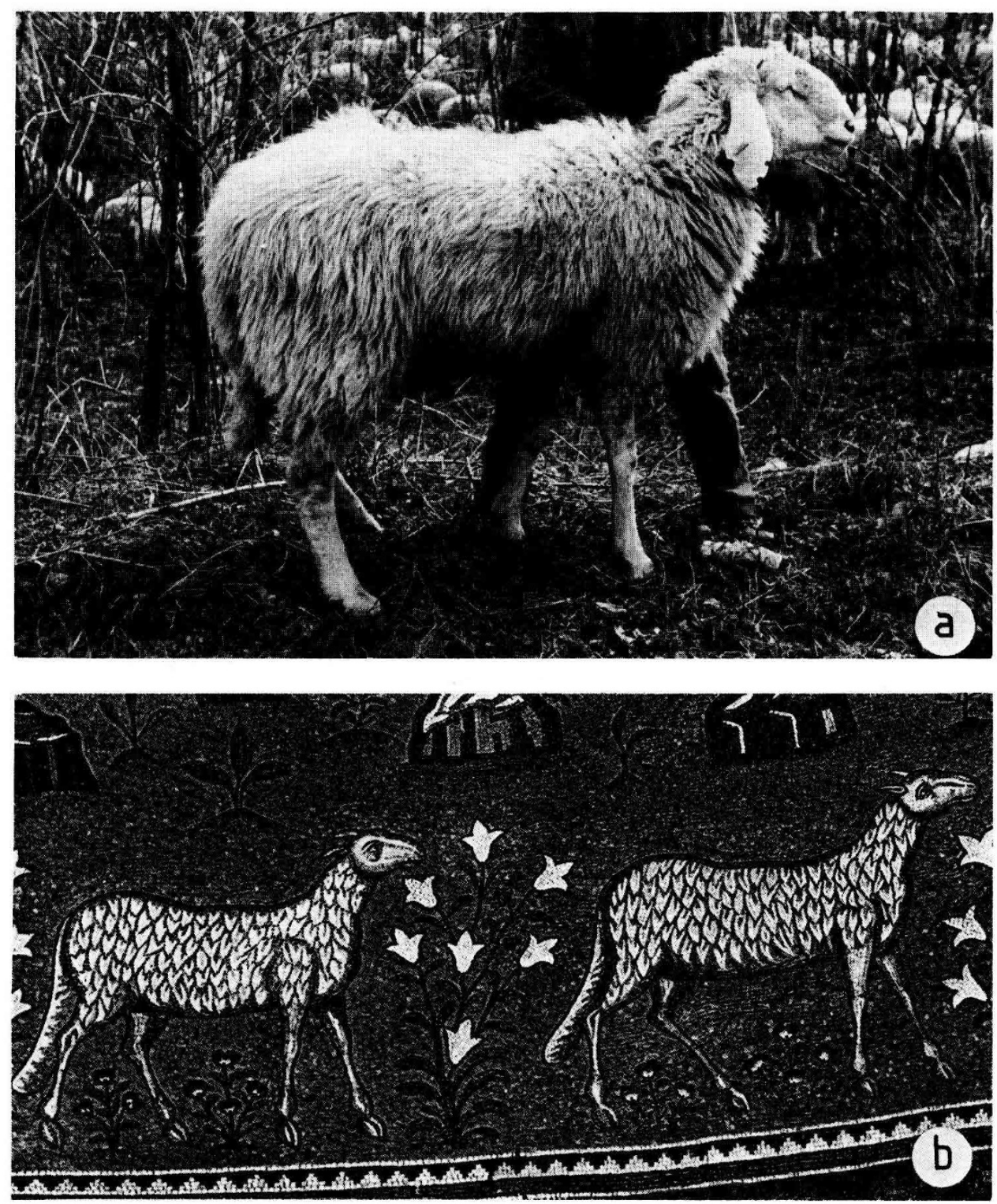

FIG. 1

Types ovins de l'Italie du Nord.

Types of sheep in northern Italy.

a) Bélier Biellese $\times$ Bergamasque : type de race influençante. Biellese $\times$ Bergamasca ram : influential breed type.

b) Mosaïque de Saint-Appolinaire en Classe Ravenne, $\mathrm{VI}^{\mathrm{e}}$ siècle : brebis blanches sans cornes, à chanfrein busqué longue queue et toison ouverte très semblables au bélier de a. White, hornless, long tailed open fleeced ewes with a roman nose on a mosaic of $S$. Appolinare en Classe, Ravenna, 6th century. They are very similar to the ram in a. 
Les lieux où coexistent des populations traditionnelles et des populations plus " évoluées » qui tendent à les remplacer, constituent autant de zones d'étude privilégiées.

Dans le cadre d'un projet finalisé du C.N.R., intitulé «Défense des ressources génétiques des populations animales »(1), une enquête sur les populations ovines et caprines d'Italie, conduite par l'un des auteurs (BonaCINI, 1980), a montré que la région de l'Arc Alpin italien pouvait constituer une de ces zones d'étude privilégiée pour les populations ovines.

Il existe en effet, dans cette région, des races ovines à effectif important qui sont utilisées dans des systèmes d'élevage orientés vers la production de masse (par exemple le Bergamasque) et dont les béliers sont plus couramment employés pour «améliorer " les populations environnantes incluses, elles, dans des systèmes plus traditionnels.

Il se trouve en outre que ces races amélioratrices que nous appellerons dans la suite de cet article "influençantes », présentent des caractéristiques extérieures qui peuvent être considérées, au moins à première vue, comme évoluées (par exemple la grande taille, le chanfrein busqué, les longues oreilles) (cf. fig. 4) par rapport à celles du mouton traditionnel ayant peuplé précédemment l'Europe alpine, comme le mouton des Grisons, maintenant disparu (EugSTER, 1921 ; MASON, 1967).

Cette particularité suggère l'utilisation de gènes et caractères à effets visibles selon une méthode proposée par LaUVergne (1978, 1979 b, 1982 b) : en mesurant au moyen d'un indice d'archaïsme le degré d'imprégnation des populations locales par les races influençantes. C'est le propos du présent article.

\section{Matériel et méthodes}

\section{A. Le cadre géographique}

L'Arc Alpin italien est limité à l'Ouest, au Nord et à l'Est par les frontières avec la France, la Suisse, l'Autriche et la Yougoslavie. Ces frontières se superposent la plupart du temps avec la ligne de partage des eaux.

Vers le Sud, nuus avons limité notre zone d'investigation à une ligne qui, partant de la Spezia va vers le Nord, Nord-Est pour s'infléchir au contact de la plaine vers le Sud-Est, de manière à exclure du domaine considéré les collines sub apennines et l'Apennin septentrional.

En simplifiant quelque peu la géographie agronomique de l'Arc Alpin italien, on peut, avec l'I.T.P.A. (1967) considérer trois régions naturelles : une plaine d'effondrement drainée par le Pô et entourée d'une zone collinaire en fer à cheval, elle-même emboîtée dans la montagne alpine (cf. la carte de la figure 2).

(1) En italien « Difesa delle risorse genetiche delle populazioni animali " (C.N.R., 1980) sous la responsabilité de l'un des auteurs (G. ROGNONI). 


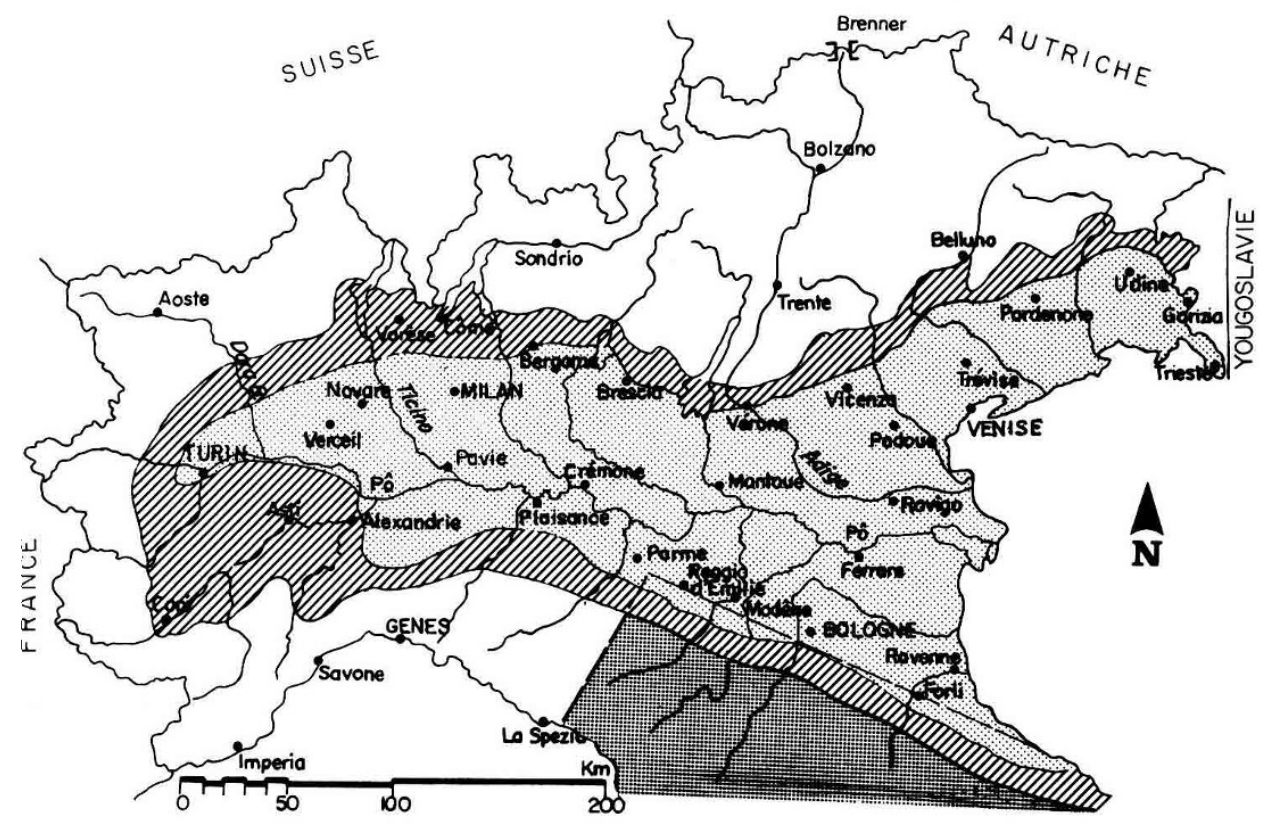

FIG. 2

Les grandes régions naturelles agronomiques de l'Arc Alpin italien.

Main agronomic natural regions of the Italian Alpine Bow.

montagne (Apennin)

एव] collines

plaine du Pô

montagne (Arc alpin)

- - limites de la zone considérée

\section{B. L'élevage ovin dans l'Arc Alpin italien}

\section{Généralités}

Comparé au reste de l'Italie, l'Arc Alpin n'est pas une grande région d'élevage ovin. Un examen des données de démographie humaine et ovine révèle en effet que, si l'Italie du Nord a une densité humaine du même ordre de grandeur que celle du pays pris dans son ensemble, par contre, les ovins y sont 9 fois moins abondants (cf. tableau 1).

Cela tient principalement au climat et au relief. La zone montagneuse alpine très arrosée pendant la belle saison se prête tout à fait à l'élevage bovin, cependant que la plaine padane, aisément irrigable, peut porter des cultures industrielles et fourragères intensives qui permettent, elles aussi, un développement de l'élevage bovin. L'élevage ovin se trouve ainsi relégué dans la zone collinaire, comme nous allons le voir en examinant de plus près les régions évoquées. 


\section{TABLEAU 1}

Données de démographie humaine et ovine pour l'Italie du Nord.

Some demographic data on humans and ovines in northern Italy.

\begin{tabular}{|c|c|c|c|c|c|c|}
\hline & \multirow{2}{*}{$\begin{array}{c}\text { Superficie } \\
\text { en }^{2} m^{2}\end{array}$} & \multirow{2}{*}{$\begin{array}{l}\text { Habitants: } \\
\text { recensement } \\
\text { de } 1971\end{array}$} & \multicolumn{2}{|c|}{ Effectifs ovins ISTAT } & \multicolumn{2}{|c|}{ Densité au km² } \\
\hline & & & 1960 & 1979 & $\begin{array}{c}\text { Humaine } \\
1971\end{array}$ & $\begin{array}{c}\text { Ovine } \\
1979\end{array}$ \\
\hline Italie & 301243 & 54964012 & 8230800 & 9864000 & 182,5 & 32,7 \\
\hline Italie du Nord & $\begin{array}{c}119846 \\
(38 \%)\end{array}$ & $\begin{array}{c}24134846 \\
(40 \%)\end{array}$ & $\begin{array}{c}459800 \\
(5,5 \%)\end{array}$ & $\begin{array}{c}446000 \\
(4,5 \%)\end{array}$ & 201,4 & 3,7 \\
\hline
\end{tabular}

\section{La plaine du Pô}

La plaine du Pô occupe une superficie d'environ $42000 \mathrm{~km}^{2}$ qui a, en gros, la forme d'un triangle isocèle de $300 \mathrm{~km}$ de base (la Côte Adriatique) et de $320 \mathrm{~km}$ de hauteur (un axe Ouest-Est parallèle au Pô) (I.S.T.A.T., 1981).

La dominance zootechnique est bovine laitière comme déjà vu, avec l'extension marquée, ces dernières années, de la race Holstein-Frisonne d'Amérique au détriment de la Brune des Alpes (I.T.P.A., 1967 ; Lauvergne, 1980 ; A.I.A., 1962 à 1981).

Dans cette région naturelle, il n'y a pas, à proprement parler, d'implantations ovines fixes, mais les troupeaux d'éleveurs établis dans la zone collinaire que nous examinerons ensuite, y séjournent volontiers en hiver.

\section{Les collines}

Cette zone, dont l'altitude varie entre 200 et $600 \mathrm{~m}$, a une largeur qui, souvent, n'excède pas $30 \mathrm{~km}$ (cf. fig. 2). La surface envisagée dans notre étude est d'environ $20000 \mathrm{~km}^{2}$.

Il s'agit d'une région pentue où les sols, moins facilement irrigables que dans la plaine padane ont, en outre, une potentialité agronomique plus faible (I.T.P.A., 1967).

Bien que, dans cette zone, l'élevage bovin soit dominant, celui des ovins prend une certaine importance. C'est là, en effet, que sont basés les éleveurs possédant la majorité des moutons de l'Arc Alpin : de 250000 à 300000 têtes selon une estimation basée sur les données de l'ASSONAPA (1972).

Un des systèmes d'élevage très courant dans la région est celui de la grande transhumance, dite aussi horizontale. En été, les animaux sont dans la montagne et l'hiver ils vont dans la plaine padane tout en transitant dans la zone collinaire pendant les saisons intermédiaires.

Ces moutons appartiennent principalement aux races que nous avons considérées plus haut comme influençantes, selon des critères d'importance numérique dans le passé et encore actuellement et les témoignages passés et actuels de diffusion de reproducteurs 
à l'entour donnés par exemple par l'atlas du F.E.I.C.A. (1967) et vérifiés lors de l'enquête de Bonacini (1980).

Ces races influençantes sont la Bergamasca (en français la Bergamasque), la Biellese, la Lamon, et la Delle Langhe, toutes races bouchères, à l'exception de la Delle Langhe qui a aussi une vocation laitière.

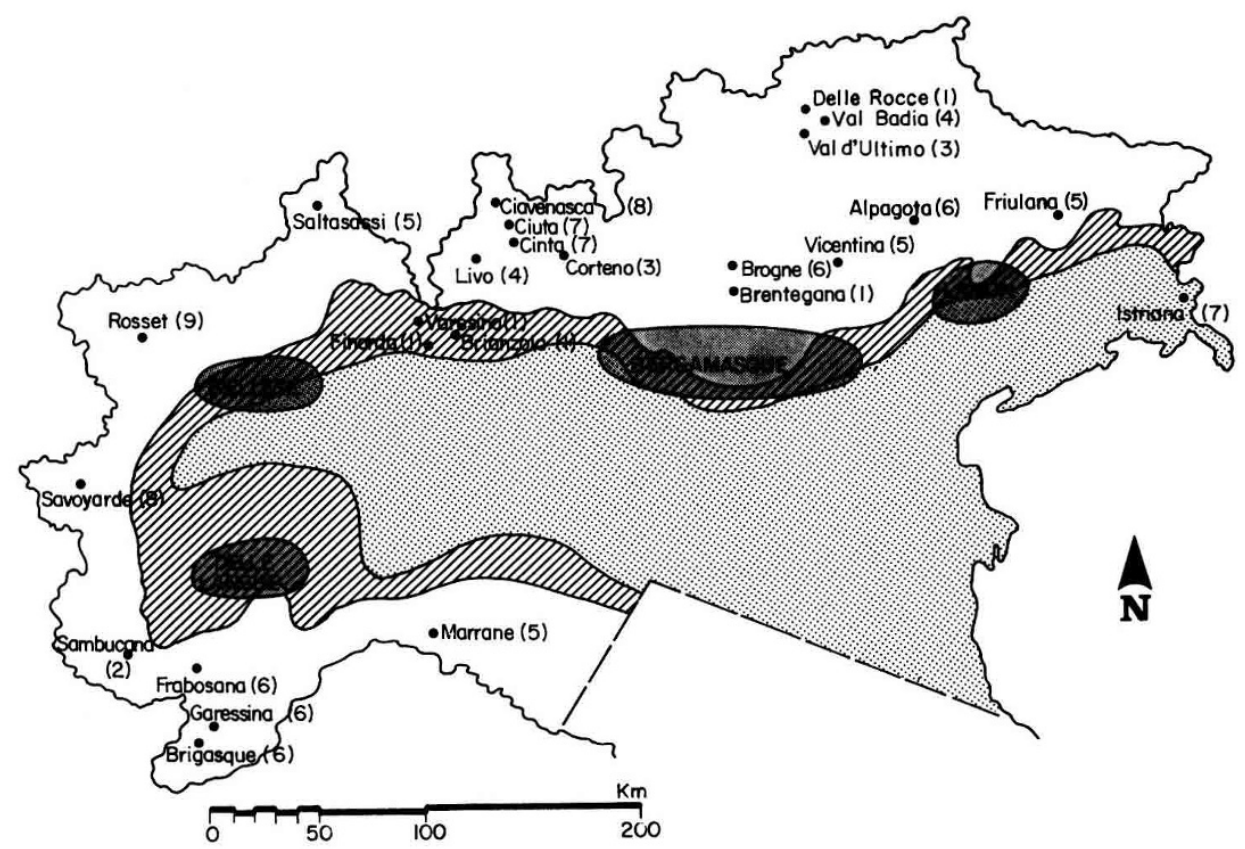

FIG. 3

Les races ovines de l'Arc Alpin italien: localisation et indice d'archaïsme.

Sheep breeds of the Italian Alpine Bow : localization and indices of archaism.

\section{La montagne}

La zone alpine couvre environ $50000 \mathrm{~km}^{2}$ (I.S.T.A.T., 1981). A côté de l'élevage bovin qui domine (avec divers systèmes et diverses races selon les zones), il y existe un petit élevage ovin autochtone complémentaire à caractère traditionnel, cependant que les pâturages d'altitude sont, au moins en partie, loués à des transhumants venus de la zone collinaire.

Les éleveurs ovins indigènes de cette zone pratiquent volontiers des remues, faites de déplacements de petite amplitude et dites parfois transhumances verticales. Ces remues s'effectuent généralement entre la vallée et les versants des monts qui la délimitent. 
OVINS DE L'ARC ALPIN ITALIEN

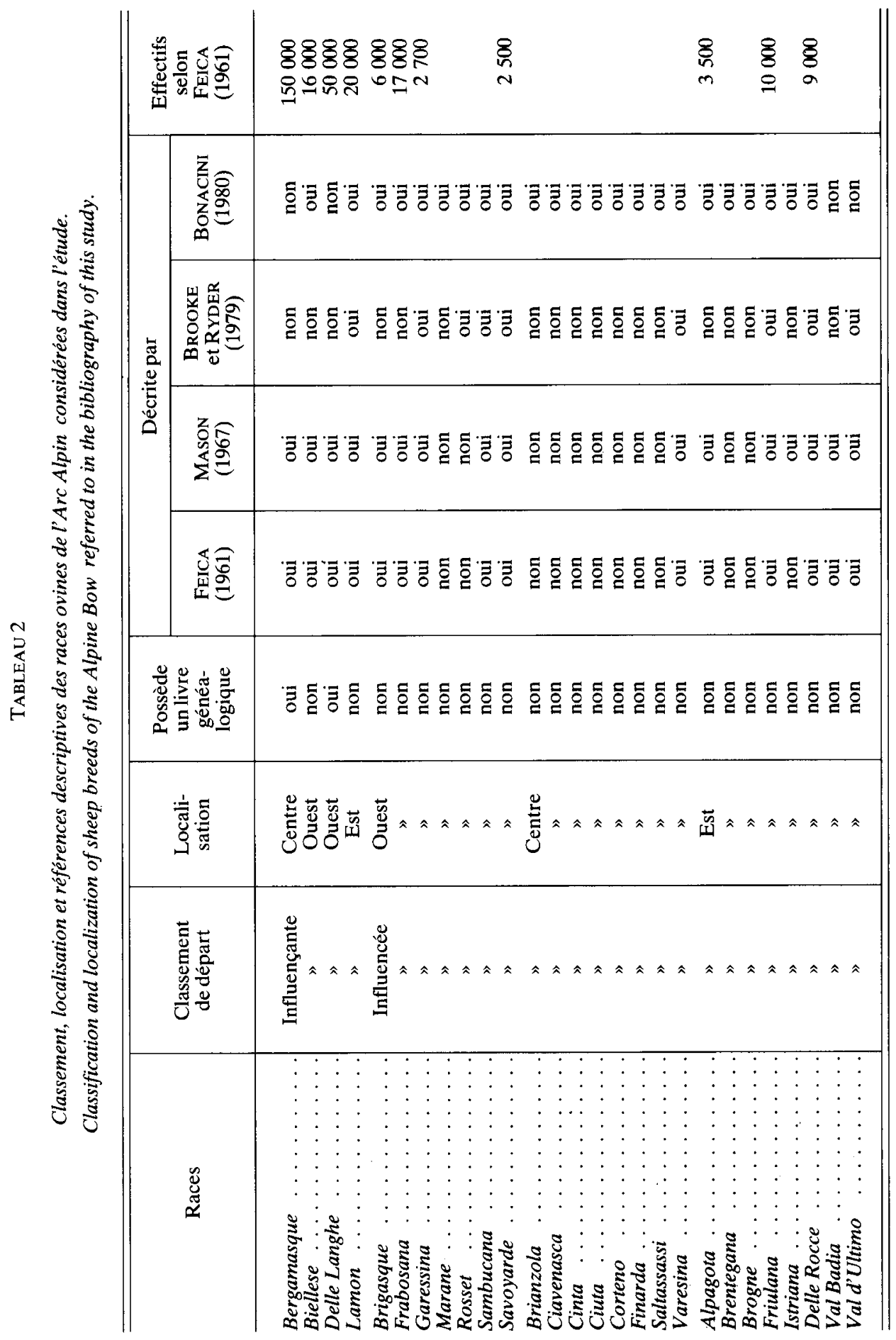


Les ovins (dont l'effectif dans cette zone est estimé entre 100000 et 150000 têtes; ASSONAPA, 1972) sont fragmentés en petites populations dont certaines portent un nom et ont le statut de races locales. Ce sont ces populations que nous considérons principalement comme les races influencées dans notre étude.

\section{Les races et populations envisagées}

L'enquête de Bonacinı (1980) a englobé 29 races ou populations dont 19 déjà décrites par de précédents auteurs (F.E.I.C.A., 1961 ; MASON, 1967 ; BROOKE \& Ryder, 1978).

Lors de l'enquête de BonaCini, les descriptions des précédents auteurs ont été vérifiées et, le cas échéant, complétées. On a adopté, pour désigner les races, la terminologie italienne sauf pour les races qui ont un nom courant en français : Bergamasque, Brigasque, Savoyarde. Pour la Delle Rocce, basée sur le Tyrol du Sud, on peut rappeler son nom allemand: Tiroler Bergschaf ou Steinschaf, nom sous lequel elle est souvent évoquée, même en France.

Dans la liste du tableau 2, où les populations ou races ont été classées en " influençantes" et "influencées", ces dernières ont été réparties en trois groupes: de l'Ouest, du Centre et du Nord.

\section{Méthode}

\section{Les principes de l'étude}

On a tout d'abord recherché dans les descriptions des races les caractères extérieurs permettant la discrimination, c'est-à-dire ceux pour lesquels existe une certaine variabilité entre races.

On a ensuite, pour ces caractères discriminants, établi une échelle d'archaïsme en trois notes.

Les notes d'archaïsmes ont ensuite été combinées en un indice d'archaïsme.

Après avoir vérifié qu'il existait bien en moyenne une différence d'indice d'archaïsme entre les races influençantes et les races influencées, on a ensuite cherché à établir une équation de régression de cet indice en fonction de l'éloignement de la race influencée par rapport à la race influençante la plus proche.

\section{La recherche des caractères discriminants et l'établissement d'un indice d'archaïsme}

Dans les descriptions des races d'où nous partons (standards donnés par la FE.I.C.A., 1961 ; Mason, 1967 ; BroOke \& Ryder, 1978 et descriptions de Bonacini, 1980) les caractères utilisables comme marqueurs génétiques à effets visibles sont au nombre de huit.

Trois d'entre eux, pour lesquels les races et populations considérées sont toutes semblables (au moins d'après les descriptions dont nous disposions), ne sont pas discriminants. Ce sont : le type de toison (toujours ouvert), le type de laine (toujours grossier) et la couleur de la toison (toujours blanche). 
Les cinq critères discriminants le format (la taille et le poids), le profil frontonasal chez la femelle (celui du mâle est beaucoup plus accentué et son accentuation non étudiée en détail semble par trop varier avec l'âge), la forme et la longueur des oreilles, le cornage et la coloration des extrémités poilues.

Pour les cinq caractères discriminants, l'échelle d'archaïsme en trois notes $(0,1$, 2 : du moins archaïque au plus archaïque) est donnée dans le tableau 3.

\section{TABLEAU 3}

Echelle d'archaïsme utilisée dans la présente étude.

Scale of archaism used in the present study.

\begin{tabular}{l|l|c|l}
\hline \multicolumn{1}{c|}{ Caractères } & \multicolumn{1}{|c|}{ Echelle } & Notes & \multicolumn{1}{c}{ Remarques } \\
\hline Format & Grande & 0 & $>60 \mathrm{~kg} ;>70 \mathrm{~cm}$ au garrot \\
& Moyenne & 1 & $50-60 \mathrm{~kg} ; 60-70 \mathrm{~cm}$ au garrot \\
& Petite & 2 & $<50 \mathrm{~kg} ;<60 \mathrm{~cm}$ au garrot \\
\hline \multirow{2}{*}{ Profil fronto-nasal } & Busqué & 0 & Cf. fig. 4 \\
& Moyennement busqué & 1 & \\
& Droit & 2 & \\
\hline \multirow{2}{*}{ Oreilles } & Longues et pendantes & 0 & Cf. fig. 4 \\
& Moyennes & 1 & \\
\hline \multirow{2}{*}{ Couleur des zones poilues } & Courtes & 2 & \\
& Tout blanc & 0 & \\
& Tacheté & 1 & \\
\hline \multirow{2}{*}{ Cornage } & Pigmenté uniforme & 2 & \\
& Absent dans les deux sexes & 0 & \\
& Eventuel & 1 & \\
\hline \hline
\end{tabular}

Dans la figure 4 cette échelle est illustrée pour le profil fronto-nasal et les oreilles.

Les justifications de cette notation de l'archaïsme sont les suivantes :

- pour le format (la taille et le poids): les moutons anciens sont de petite taille (ZEUNER, 1963);

- pour le profil fronto-nasal : le chanfrein droit est une caractéristique archaïque (SERRA, 1948) ;

- pour la forme des oreilles: le mouton ancien a des oreilles petites et dressées (SERRa, 1948) ;

- pour la couleur des zones poilues (tête et pattes): les populations les plus archaïques présentent un polymorphisme coloré considérable, par exemple les races 

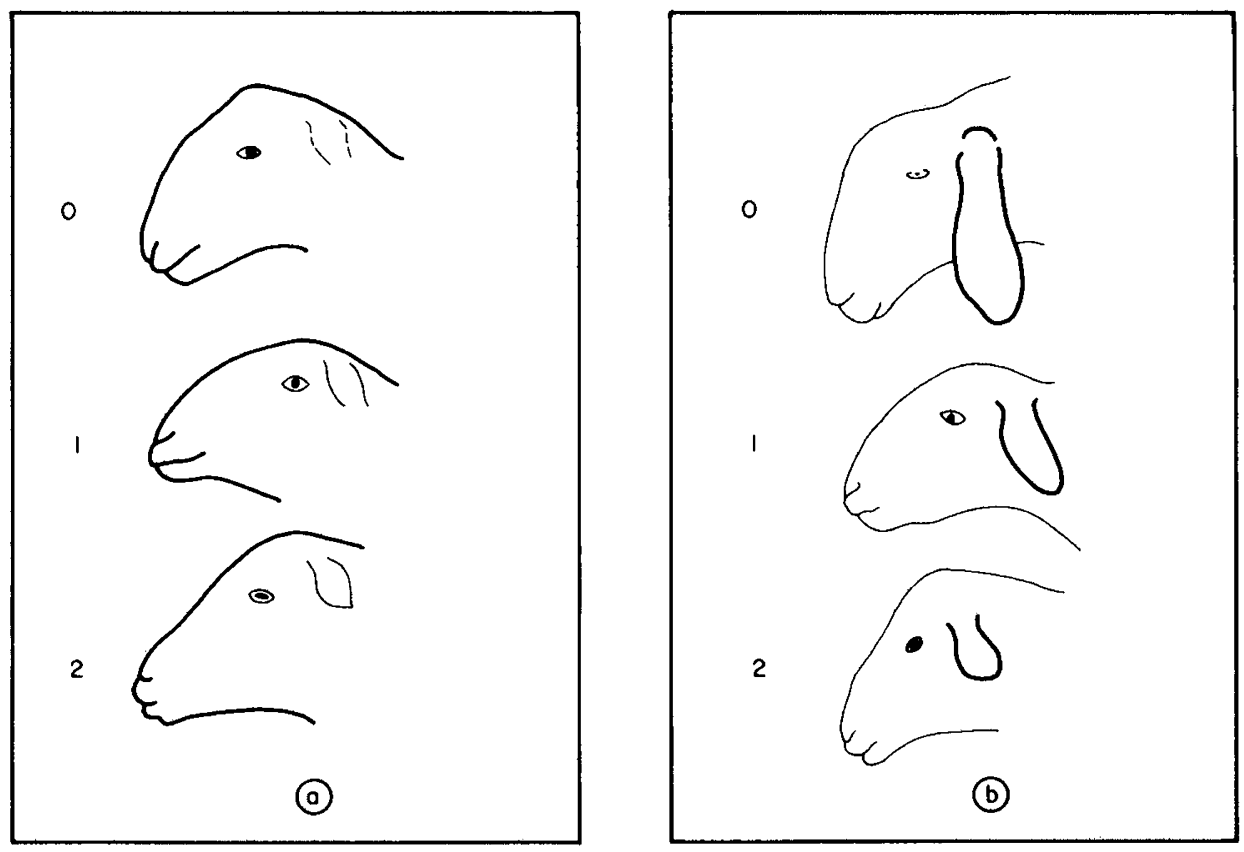

FIG. 4

L'échelle de cotation de l'archaïsme pour le profil fronto nasal et les oreilles de la présente étude. Archiac rating of head profile and ears in the present study.

de l'Atlantique Nord et de la Corse (Adalsteinsson \& Wardum, 1978) de la Norvège ancienne (BERGE, 1958) et du Cameroun (MASON, 1977). Par la suite, les races améliorées ont eu une toison blanche, un stade intermédiaire étant la persistance de la coloration sur les zones poilues périphériques;

- pour le cornage: dans l'espèce sauvage, les mâles sont cornus, les femelles sont " cornettes » ou acérates (PFEFFER, 1967). Le stade d'acératie pour les deux sexes est une acquisition récente (SERRA, 1948).

L'indice d'archaïsme (i) utilisé dans la suite de l'étude a été simplement la somme arithmétique non pondérée des notes d'archaïsme pour les cinq caractères discriminants.

Cet indice peut varier entre zéro (le moins archaïque) et dix (le plus archaïque).

\section{Résultats}

\section{A. L'indice d'archaïsme des races de l'Arc Alpin}

Dans le tableau 4, les notes et l'indice d'archaïsme sont donnés pour les 29 races considérées dans l'étude. Dans la carte de la figure 3, ces races sont reportées avec leur localisation et leur indice d'archaïsme. 
TABLEAU 4

Notes et indices d'archaïsme des races ovines de l'Arc Alpin considérées dans l'étude.

Archaic rating and indices of sheep breeds of the Alpine Bow in this study.

\begin{tabular}{|c|c|c|c|c|c|c|c|}
\hline \multirow[b]{2}{*}{ Classement } & \multirow[b]{2}{*}{ Races } & \multicolumn{5}{|c|}{ Notes d'archaïsme } & \multirow[b]{2}{*}{$\begin{array}{c}\text { Indice } \\
\text { d'archaïsme }\end{array}$} \\
\hline & & Format & $\begin{array}{c}\text { Profil } \\
\text { fronto- } \\
\text { nasal }\end{array}$ & Oreilles & $\begin{array}{c}\text { Couleur } \\
\text { tête et } \\
\text { pattes }\end{array}$ & Cornage & \\
\hline Influençantes & $\begin{array}{l}\text { Bergamasque . . . . } \\
\text { Biellese ... . . . . } \\
\text { Delle Langhe . . . } \\
\text { Lamon . . . . . }\end{array}$ & $\begin{array}{l}0 \\
0 \\
1 \\
0\end{array}$ & $\begin{array}{l}0 \\
0 \\
0 \\
0\end{array}$ & $\begin{array}{l}0 \\
0 \\
1 \\
0\end{array}$ & $\begin{array}{l}0 \\
0 \\
0 \\
2\end{array}$ & $\begin{array}{l}0 \\
0 \\
0 \\
0\end{array}$ & $\begin{array}{l}0 \\
0 \\
2 \\
2\end{array}$ \\
\hline $\begin{array}{c}\text { Influencées } \\
\text { (Ouest) }\end{array}$ & 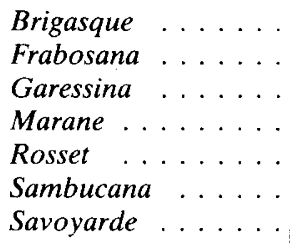 & $\begin{array}{l}1 \\
1 \\
2 \\
2 \\
2 \\
1 \\
1\end{array}$ & $\begin{array}{l}0 \\
0 \\
1 \\
1 \\
2 \\
0 \\
1\end{array}$ & $\begin{array}{l}1 \\
2 \\
2 \\
1 \\
2 \\
1 \\
2\end{array}$ & $\begin{array}{l}2 \\
2 \\
1 \\
1 \\
2 \\
0 \\
2\end{array}$ & $\begin{array}{l}2 \\
1 \\
0 \\
0 \\
1 \\
0 \\
2\end{array}$ & $\begin{array}{l}6 \\
6 \\
6 \\
5 \\
9 \\
2 \\
8\end{array}$ \\
\hline $\begin{array}{l}\text { Influencées } \\
\text { (Centre) }\end{array}$ & 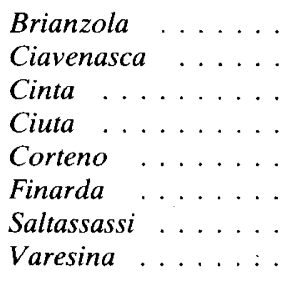 & $\begin{array}{l}0 \\
2 \\
2 \\
2 \\
1 \\
0 \\
2 \\
0\end{array}$ & $\begin{array}{l}1 \\
2 \\
2 \\
2 \\
1 \\
1 \\
2 \\
1\end{array}$ & $\begin{array}{l}0 \\
2 \\
2 \\
2 \\
1 \\
0 \\
1 \\
0\end{array}$ & $\begin{array}{l}0 \\
1 \\
0 \\
0 \\
0 \\
0 \\
0 \\
0\end{array}$ & $\begin{array}{l}0 \\
1 \\
1 \\
1 \\
0 \\
0 \\
0 \\
0\end{array}$ & $\begin{array}{l}1 \\
8 \\
7 \\
7 \\
3 \\
1 \\
5 \\
1\end{array}$ \\
\hline $\begin{array}{l}\text { Influencées } \\
\text { (Est) }\end{array}$ & 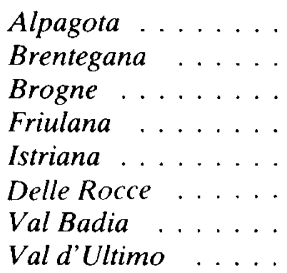 & $\begin{array}{l}2 \\
0 \\
2 \\
1 \\
1 \\
0 \\
2 \\
1\end{array}$ & $\begin{array}{l}1 \\
0 \\
1 \\
2 \\
0 \\
1 \\
1 \\
1\end{array}$ & $\begin{array}{l}1 \\
0 \\
1 \\
1 \\
2 \\
0 \\
1 \\
1\end{array}$ & $\begin{array}{l}2 \\
1 \\
2 \\
1 \\
2 \\
0 \\
0 \\
0\end{array}$ & $\begin{array}{l}0 \\
0 \\
0 \\
0 \\
2 \\
0 \\
0 \\
0\end{array}$ & $\begin{array}{l}6 \\
1 \\
6 \\
5 \\
7 \\
1 \\
4 \\
3\end{array}$ \\
\hline
\end{tabular}

B. Différence de l'indice d'archaïme moyen entre les races influencées et influençantes

Les 4 races influençantes considérées ici ont un indice d'archaïsme moyen $\overline{1}=1$. Celui des 25 races influencées étant $\bar{i}=4,32$. Comme $t=2,32$ avec une probabilité d'atteindre une valeur supérieure comprise entre 0,05 et 0,025 , on peut donc admettre que ces deux groupes de races diffèrent bien en moyenne. Cela nous autorise donc à poursuivre le cours de notre analyse, comme projeté.

\section{L'indice d'archaïsme des races influencées en fonction de l'éloignement d'avec les races influençantes}

Sur la figure 5 on a représenté l'indice d'archaïsme des populations dites influencées en fonction de l'éloignement de la race influençante la plus proche (abscisses, en $\mathrm{km}$ ). 


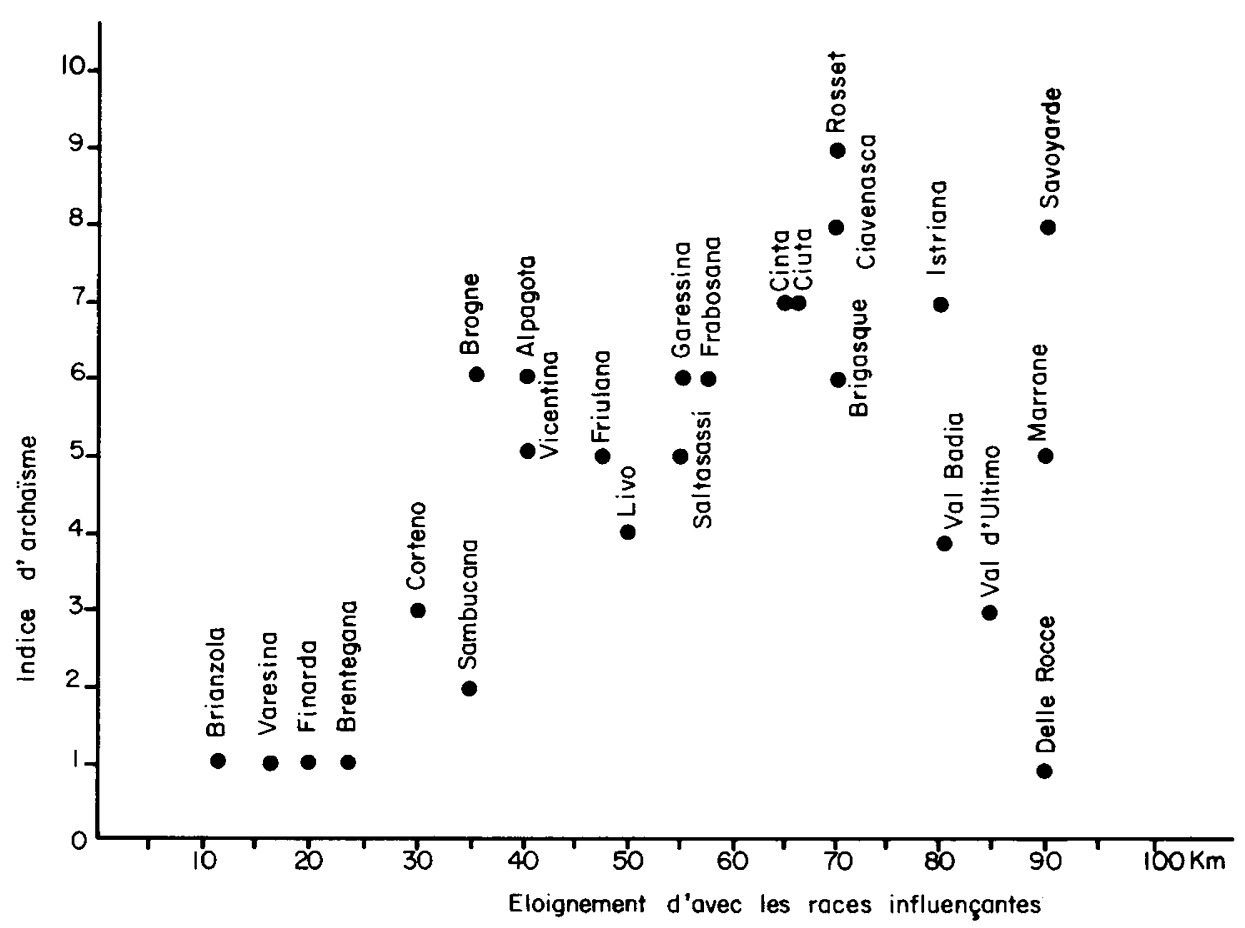

FIG. 5

Distribution des races ovines influencées selon leur indice d'archaïsme et leur éloignement des races influençantes.

Distribution of influenced sheep breed according to indice of archaism and distance from an influential breed.

Dans le tableau 5 on a testé les diverses possibilités d'une régression : linéaire, carrée, cubique, en considérant tout d'abord toutes les races influencées (ligne 1), puis en faisant abstraction des données déviantes de la figure $4:$ tout d'abord celles du groupe Nord (Delle Rocce, Val Badia, Val d'Ultimo) (ligne 2), puis retranchant en outre la Marane (ligne 3). Dans la ligne 4, on a réintroduit la Val Badia et la Val d'Ultimo mais en mesurant leur éloignement non pas d'avec une des 4 races influençantes considérées dans notre éloignement mais d'avec la Delle Rocce, consacrée race influençante.

Il se trouve en effet que ces 4 races se placent en dehors du nuage de points de la figure 5 et qu'il faudra trouver une explication spéciale à ce comportement-là.

En faisant déjà abstraction de trois de ces races (ligne 2 du tableau 5) la valeur de $\mathrm{R}^{2}$. s'élève de 0,052 à 0,208 pour le premier degré et ne diffère alors plus guère de celles de $R^{2}$ pour le second et troisième degré rendant inutile l'utilisation d'une équation de régression autre que linéaire alors que, en considérant toutes les races influencées de départ il fallait au moins prendre l'équation du deuxième degré. 


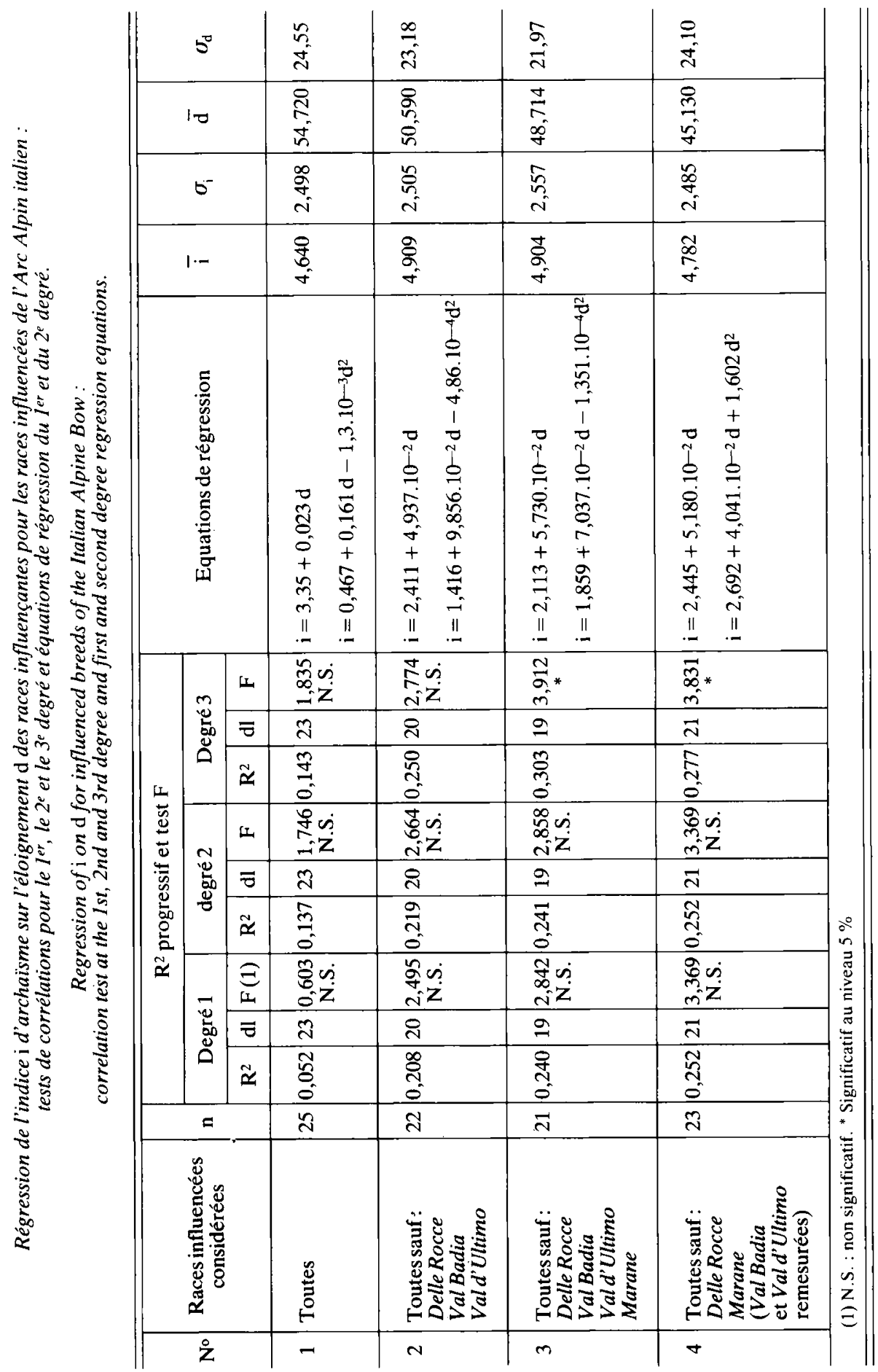




\section{Discussion}

\section{A. Les imperfections de l'étude}

\section{Dans les caractères utilisés et leur mesure}

Les critères utilisés sont ceux utilisés en zootechnie spéciale traditionnelle. Pour notre propos, il manque, entre autres choses, l'éniumération, avec leur fréquence, des variants colorés qui ségrègent encore. En outre, on ne connaît que très approximativement la structure de la toison et ses variations intra populations. Il se pourrait bien, en effet que, dans les races traditionnelles, il y ait une gamme plus large de types de toison que dans les races collinaires. On pourrait, par ce type d'examen de la variation visible, faire mieux intervenir ce facteur d'archaïsme qu'est la variabilité même d'un caractère, variabilité trop souvent occultée par des examens hâtifs avec l'idée préconçue que toutes les races ou populations ont un standard univoque.

\section{Dans l'évaluation des distances entre race influencée et race influençante la plus proche}

Cette évaluation dépend en effet de la localisation du centre de gravité de la race influencée et de la zone d'extension de la race influençante. Si certaines localisations ou limites sont fixées depuis longtemps, d'autres ont été beaucoup plus fluctuantes dans le passé et le sont encore actuellement d'où l'introduction d'un certain facteur de subjectivité.

\section{Dans la classification entre races influençantes et influencées}

L'examen de la figure 5 a révélé un certain nombre de deviants. Ceux d'un groupe Nord (Delle Rocce, Val Badia, Val d'Ultimo) s'expliquent par le fait qu'il faut plutôt considérer le Delle Rocce comme une race influençante que comme une race influencée. La vallée de l'Adige qui conduit au Brenner, est si importante comme voie de passage qu'elle doit être considérée comme capable d'abriter des races ovines influençantes, au même titre que la zone collinaire en fer à cheval et au contraire des nombreuses vallées alpestres étroites qui finissent en cul-de-sac et n'abritent que des populations traditionnelles.

L'exception de la Marane au Sud pourrait également s'expliquer par sa localisation sur un axe de passage, de l'Arc Alpin vers le Sud, cette fois.

\section{B. La vérification dès hypothèses de départ}

Quelles que soient les imprécisions que nous venons de voir, il n'en reste pas moins que la figure 5 expliquée par les équations du tableau 5 non seulement permet de vérifier la justesse des hypothèses de départ, mais donne un début de mesure objective du phénomène de recouvrement d'une population par une autre. Il s'agit pour le moment d'une simple photographie à un instant $t$ d'un phénomène qui a débuté à une époque non précisée et selon des modalités à définir, mais on perçoit bien qu'on est en présence d'un processus du même type que celui que LAUVERGNe (1978, $1979 \mathrm{a}$, b) a proposé pour expliquer la répartition primitive des ovins domestiques dans l'Ancien Monde : à partir d'un centre de différenciation et de dispersion, qui, par son dynamisme, a imposé sa loi génétique à l'entour. 
Dans le cas de l'Italie du Nord, le "centre» a une disposition en fer à cheval et son action de recouvrement ne s'exerce pas dans toutes les directions du plan, mais le schéma fondamental reste bien le même.

\section{Le gradient de l'indice d'archaïsme fonction de la distance}

D'après la ligne 4 du tableau 5, on voit que l'indice d'archaïsme croît d'environ 1 point tous les $20 \mathrm{~km}$.

\section{Le scénario du peuplement ovin de l'Italie du Nord}

Même encore en l'absence de repères démographico-génétiques précis dans le passé, on est tenté de proposer un scénario de peuplement ovin de l'Italie du Nord au cours des derniers siècles.

On peut penser tout d'abord que l'ensemble du pays a été peuplé très anciennement comme toute l'Europe occidentale (ZEUNER, 1963) par un animal sans doute peu différent de l'actuel mouton Islandais ou Norvégien ancien, comme en témoigne l'aspect qu'avait le mouton du canton suisse proche des Grisons qui existait encore il y a quelques années et qui est évoqué plus haut: un mouton de petite taille à oreilles dressées, chanfrein droit, queue fine longue ou mi-longue, toison très variable dans sa structure et surtout sa couleur.

Par la suite, venu sans doute d'Orient, est apparu en Italie, comme dans d'autres endroits (en particulier en Afrique, cf. Zeuner, 1963), un mouton de grande taille, à chanfrein busqué, oreilles longues et tombantes, queue longue et fine. C'est ce mouton, appelé parfois par les auteurs italiens Ovis aries Sudanica, dont la présence est attestée dès le $V^{e}$ siècle (cf. certaines mosaiques de Ravenne, fig. 16) qui a dû être pris pour créer, à partir du XIV $\mathrm{XV}^{\mathrm{e}}$ siècle certaines populations de l'Arc Alpin orientées vers la production spécialisée de laine pour une industrie lainière alors concentrée dans l'Italie du Nord (BATICLE, 1982). Cet auteur nous dit également qu'à l'époque, la grande transhumance était déjà installée dans plusieurs zones en Italie.

Il faudra attendre la naissance de la littérature zootechnique moderne, à la fin $\mathrm{XIX}^{\mathrm{e}}$ siècle et surtout au $\mathrm{XX}^{\mathrm{e}}$ siècle, pour voir nommées et décrites des races dont la création remonte alors à plusieurs siècles en arrière et qui ont subi ensuite une mérinisation plus marquée dans le Centre et le Sud de l'Italie, dans la perspective d'obtenir des tonnages importants de laine fine qui se vendait mieux, jusqu'à ce que cette spéculation zootechnique soit rendue beaucoup moins intéressante avec l'apparition des laines du Nouveau Monde et de l'Australie. Reconverties vers les productions laitières et surtout bouchères, ces races de première et de seconde générations pour la production lainière se sont néanmoins maintenues dans la zone collinaire nord-italienne tout en continuant à influencer génétiquement les populations locales qui ont opposé, cependant, quelque résistance puisqu'un certain nombre de caractères archaïques y sont encore décelables.

Dans cette perspective, il y aurait tout lieu de penser que les moutons de montagne autrichiens, Steinschaf et Bergschaf, doivent leur aspect extérieur, en particulier le chanfrein et le port des oreilles, à une influence italienne venue par le Brenner. Certains auteurs arrivent, d'ailleurs au moins implicitement, à cette conclusion (Mason, 1967). 


\section{Remerciements}

Le manuscrit de cet article a été lu par R. Laurans, Jacqueline Vu Tien et G. Baglione qui ont suggéré une série d'utiles améliorations.

Les calculs du tableau 5 ont été effectués sur un ordinateur WANG 2200 par J. LEFEBVRE.

\section{Abréviations utilisées dans le texte et la bibliographie}

A.I.A : Associazione italiana Allevatori (Rome).

ASSONAPA : Associazione nazionale della Pastorizia (Rome).

C.N.R. : Consiglio nationale delle Ricerche (Rome).

FE.I.C.A. : Federazione italiana del Consorzi Agrari (Rome).

I.S.T.A.T. : Istituto Centrale di Statistica (Rome).

I.T.P.A. : Istituto di Tecnica e propaganda agraria (Rome).

\section{Summary}

A study of the genetic profile of sheep of the Italian Alpine Bow using visible-effect markers

The Italian Alpine Bow may be divided into three main natural regions : the Pô valley, subalpine hills and mountains.

Most of the sheep (250 to 300000 head) are located in the hill region and are submitted to transhumance between the Pô valley (in winter) and mountain pastures (in summer).

In the mountains ( 100 to 150000 head), small less mobile populations are settled.

There are no sheep in the Pô valley.

According to their consistance and their anciently attested genetic influence, all four breeds - Delle Langhe, Biellese, Bergamasca and Lamon - have been considered as "influential ". In this hypothesis, the remaining 25 breeds are considered as influenced by these four breeds.

An indice of archaism, i.e. the sum of archaic ratings affecting five characters - size, head profile, length of ears, coloration of hairy parts and horns - has been determined for all the breeds. This indice varied between 0 and 10 .

The mean indice of archaism of the four influential breeds was $\bar{i}=1$, while that of the 25 influenced breeds was $\bar{i}=4.32$ and the Delle Rocce (Tiroler Bergschaf) breed must be considered as an influential breed and not as an influenced one as at the beginning.

The indice of archaism of the influenced breeds increased 1 point per $20 \mathrm{~km}$ of distance from the zone of an influential breed.

These influential breeds could have been created already in the 14th century in order to produce the wool required by the developing textile industry of northern Italy. The external criteria adopted (big size, white fleece, long ears) were then different from those of the anciently established population which have since been graded up. The incomplete disappearance of some archaic characteristics could be due to the fact that a subsistance breeding system has been retained in some alpine valleys. 


\section{Références bibliographiques}

AdALSTEINSSON S., WARDUM H., 1978. Frequency of color genes in Faeroe Islands Sheep. J. Hered., 69, 259-262.

A.I.A., 1962 à 1981. Bolletini dei controlli funzionali, Roma.

ASSONAPA, 1972. Indagine nazionale su alcuni aspetti degli allevamenti e delle produzione ovine. N.2. Allevamenti abitazioni, ricoveri. Ramo editoriale degli agricoltori, Roma, 55 p.

Baticle Y., 1982. La Laine. Masson, Paris, 259 p.

BERGE S., 1958. Farger hos den gamle Norske sauen (couleur du mouton Norvégien Ancien). Meld. Norges Landbrukhøgsk., 6, 19 p.

BonaCINI I., 1980. Razze e popolazioni ovine e caprine italiane. In : Progetto Finalizzato "Difesa delle Risorse Genetiche delle popolazioni animale ». C.N.R., Roma (sous presse).

BROOKE C.-H., RYDER M.-L., 1979. Races ovines méditerranéennes en régression. O.N.U.A.A. et P.N.U.E., Rome, 63 p.

C.N.R., 1980. Le Risorse Genetiche animali, conservazione e utilizzo. C.N.R. Roma, 222 p.

EUGSTER G., 1921. Beiträge zur Monographie Bündner - Oberländerschafes. Inaug. Dissert. Universität Bern, $108 \mathrm{p}$.

F.E.I.C.A., 1961. Atlanti di Zootecnica. Allevamenti italiani. 2. Ovini. Ramo editoriale degli agricoltori, Roma, $225 \mathrm{p}$.

I.S.T.A.T., 1981. Bolletini annuali e "Le Regione in cifré ", Roma.

I.T.P.A., 1967. Petit guide de l'Agriculture italienne. Istitu di Tecnica e Propaganda Agraria, Rome, $514 \mathrm{p}$.

LAUVERGNe J.-J., 1978. Utilisation des marqueurs génétiques pour l'étude de l'origine et de l'évolution du mouton domestique. Ethnozootechnie, 27, 17-23.

LAuvergne J.-J., 1979 a. Modèles de répartition des populations domestiques animales après migration par vagues à partir d'un centre d'origine. Ann. Génét. Sél. anim., 11, 105-110.

LAUVERGNE J.-J., $1979 \mathrm{~b}$. Visible genetic markers in domestic sheep and their ancestors. In : FoOTE W.C., Bunch T.D. The Domestication of Sheep. International Sheep and Goat Institute, Logan, Utah, 21-23.

LAUVERGNe J.-J., 1980. Dynamique des populations élevées en Europe dans la zone méditerranéenne. In : Le risorse genetiche animali, conservazione e utilizzo, C.N.R., Rome, 159-170.

LAUVERGNE J.-J., 1981. L'organisation de la conservation et de la gestion des stocks génétiques pour les gros animaux de ferme. In: Animal genetic resources, F.A.O., Animal Production and Health paper $n^{\circ} 24$, Rome, 318-331.

LAUVERGNE J.-J., 1982 a. Genetica en problaciones animales despues de la domesticación : consecuencias para la conservación de las razas. II Congr. mund. Genet. appl. Prod. Ganad., Madrid, 6, 77-87.

LAUVERGNE J.-J., 1982 b. Gènes marqueurs, indice d'archaïsme génétique et modèles de migration des populations animales après leur domestication : le cas du mouton au Moyen-Orient, en Europe, et en Afrique. 4th Int. Conf. Int. Counc. Archaezool., London (sous presse).

MASON I.L., 1967. Sheep breeds of the Mediterranean. F.A.O. and C.A.B. Rome and Farnham Royal Bucks, England.

MASON I.L., 1977. Tropical sheep in Southern Cameroon. Draft report to F.A.O./U.N.E.P. in the frame of the Project Conservation of Animal Genetic Resources. 4 p. ronéoté.

Pfeffer P., 1967. Le mouflon de Corse (Ovis ammon musimon, Schreber, 1782): position systématique, écologie et éthologie comparées. Mammalia, 31 (suppl.), 262 p.

RYDER M.L., 1981. A survey of European primitive breeds of sheep. Ann. Génét. Sél. anim., 13, 381-418.

Serra J.-A., 1948. Génétique du mouton. Publ. Junta Pecuar Lisboa (Ser. A), 1, 200 p.

ZEUNER F.E., 1963. A history of domesticated animals. Hutchinson, London. 